\title{
Unfamiliar face matching with photographs of infants and children
}

\author{
Robin S S Kramer ${ }^{\text {Corresp., }}{ }^{1}$, Jerrica Mulgrew ${ }^{2}$, Michael G Reynolds ${ }^{2}$ \\ ${ }^{1}$ School of Psychology, University of Lincoln, Lincoln, United Kingdom \\ 2 Department of Psychology, Trent University, Peterborough, Ontario, Canada \\ Corresponding Author: Robin S S Kramer \\ Email address: remarknibor@gmail.com
}

Background. Infants and children travel using passports that are typically valid for five years (e.g., Canada, United Kingdom, United States, Australia). These individuals may also need to be identified using images taken from videos and other sources in forensic situations including child exploitation cases. However, few researchers have examined how useful these images are as a means of identification. Methods. We investigated the effectiveness of photo identification for infants and children using a face matching task, where participants were presented with two images simultaneously and asked whether the images depicted the same child or two different children. In Experiment 1, both images showed an infant ( $<1$ year old), whereas in Experiment 2, one image again showed an infant but the second image of the child was taken at 4-5 years of age. In Experiments 3a and $3 b$, we asked participants to complete shortened versions of both these tasks (selecting the most difficult trials) as well as the short version Glasgow face matching test. Finally, in Experiment 4, we investigated whether information regarding the sex of the infants and children could be accurately perceived from the images. Results. In Experiment 1, we found low levels of performance ( $72 \%$ accuracy) for matching two infant photos. For Experiment 2, performance was lower still (64\% accuracy) when infant and child images were presented, given the significant changes in appearance that occur over the first five years of life. In Experiments $3 a$ and $3 b$, when participants completed both these tasks, as well as a measure of adult face matching ability, we found lowest performance for the two infant tasks, along with mixed evidence of within-person correlations in sensitivities across all three tasks. The use of only same-sex pairings on mismatch trials, in comparison with random pairings, had little effect on performance measures. In Experiment 4, accuracy when judging the sex of infants was at chance levels for one image set and above chance (although still low) for the other set. As expected, participants were able to judge the sex of children (aged 4-5) from their faces.

Discussion. Identity matching with infant and child images resulted in low levels of 
performance, which were significantly worse than for an adult face matching task. Taken together, the results of the experiments presented here provide evidence that child facial photographs are ineffective for use in real-world identification. 
1 Unfamiliar face matching with photographs of infants and children

2

3 Robin S. S. Kramer ${ }^{1,2}$, Jerrica Mulgrew ${ }^{2}$, Michael G. Reynolds ${ }^{2}$

4

$5{ }^{1}$ School of Psychology, University of Lincoln, Lincoln, United Kingdom

$6 \quad{ }^{2}$ Department of Psychology, Trent University, Peterborough, Ontario, Canada

7

8 Corresponding Author:

9 Robin Kramer

10 School of Psychology, University of Lincoln, Lincoln LN6 7TS, United Kingdom

11 Email address: remarknibor@gmail.com

12

13 


\section{Abstract}

15

16 Background. Infants and children travel using passports that are typically valid for five years

17 (e.g., Canada, United Kingdom, United States, Australia). These individuals may also need to be identified using images taken from videos and other sources in forensic situations including child exploitation cases. However, few researchers have examined how useful these images are as a means of identification.

Methods. We investigated the effectiveness of photo identification for infants and children using

22 a face matching task, where participants were presented with two images simultaneously and 23 asked whether the images depicted the same child or two different children. In Experiment 1, 24 both images showed an infant $(<1$ year old), whereas in Experiment 2, one image again showed 25 an infant but the second image of the child was taken at 4-5 years of age. In Experiments $3 a$ and $263 \mathrm{~b}$, we asked participants to complete shortened versions of both these tasks (selecting the most 27 difficult trials) as well as the short version Glasgow face matching test. Finally, in Experiment 4, 28 we investigated whether information regarding the sex of the infants and children could be 29 accurately perceived from the images.

Results. In Experiment 1, we found low levels of performance (72\% accuracy) for matching two 31 infant photos. For Experiment 2, performance was lower still (64\% accuracy) when infant and 32 child images were presented, given the significant changes in appearance that occur over the first

33 five years of life. In Experiments $3 \mathrm{a}$ and 3b, when participants completed both these tasks, as 34 well as a measure of adult face matching ability, we found lowest performance for the two infant 35 tasks, along with mixed evidence of within-person correlations in sensitivities across all three 36 tasks. The use of only same-sex pairings on mismatch trials, in comparison with random 
37 pairings, had little effect on performance measures. In Experiment 4, accuracy when judging the

38 sex of infants was at chance levels for one image set and above chance (although still low) for

39 the other set. As expected, participants were able to judge the sex of children (aged 4-5) from

40 their faces.

41 Discussion. Identity matching with infant and child images resulted in low levels of

42 performance, which were significantly worse than for an adult face matching task. Taken

43 together, the results of the experiments presented here provide evidence that child facial

44 photographs are ineffective for use in real-world identification.

45
Research has repeatedly shown that deciding whether two different face photographs are of the same person, or whether a person standing in front of you is the same person depicted in a photograph, results in rapid and accurate assessments for familiar faces (Bruce, Henderson, Newman, \& Burton, 2001) and inaccurate assessments for unfamiliar faces (Bruce et al., 2001; Bruce et al., 1999; Kemp, Towell, \& Pike, 1997; Megreya \& Burton, 2006, 2008). Indeed, a benchmark test of unfamiliar face matching found performance levels of around 90\% (Burton, White, \& McNeill, 2010), representing a 'best case' scenario since images were high quality and taken only minutes apart. This detriment with unfamiliar face matching has important implications for real-world professions (e.g., border control situations) and theories of face perception. It is worth noting, for example, that passport officers are no better than the general population on such tasks (White, Kemp, Jenkins, Matheson, \& Burton, 2014). 
The focus of the present paper is the accuracy of matching infant identities using faces.

60 Face matching research has concentrated on adult faces, and to our knowledge, there has been

61 little consideration of how difficult this task may be with infants. This is surprising, given the

62 practical implications of validating the identity of an infant. Consider the issue of identifying

63 children in border control situations. It is estimated that up to 400,000 children are trafficked

64 across international borders annually (U.S. Department of State, 2007). Furthermore, baby-

65 selling and illegal adoption have been reported in Europe, Africa, Central and South America,

66 Central Asia and East Asia (UNODC, 2016). In many countries, infants are required to travel

67 using their own passports as photographic ID in order to combat trafficking, and so it is

68 important to determine the efficacy with which infants can be identified using these images.

69 The goal of the present paper is to examine whether there is an empirical reason to treat

70 infant face matching as different than adult face matching. Based on related research examining

71 how people recognise previously seen faces, we hypothesise that infant face matching will be

72 noticeably harder than adult matching. For instance, we know that viewers show an own-age bias

73 when recognising faces, where people are better at recognising previously seen faces of one's

74 own age group (Rhodes \& Anastasi, 2012), with adults showing worse recognition of previously

75 seen infants based on their faces (Chance, Goldstein, \& Andersen, 1986). This bias seems to be

76 at least partially based on experience (Harrison \& Hole, 2009), although the quality of exposure

77 to infant faces may be more important than the quantity (Yovel et al., 2012). While researchers

78 have yet to consider the possibility of such a bias when matching identities based on faces, it is

79 likely to be a problem given that infant faces appear more alike than adult faces for adult viewers

80 (based on subjective ratings; Chance et al., 1986). For the analogous situation with own- versus

81 other-race faces, evidence has shown that an own-race bias is present in both face recognition 
82 (Meissner \& Brigham, 2001) and matching tasks (Megreya, White, \& Burton, 2011; Meissner,

83 Susa, \& Ross, 2013). This suggests that performance may show the same detriment as with any

84 other group with which we have little experience. In addition, it is possible that children's faces

85 are simply more homogeneous than adult faces. For example, craniofacial shape cues to an

86 identity's sex are more pronounced after puberty (Enlow, 1982), resulting in prepubescent

87 children displaying less between-face variability. If this is the case, we should expect a serious

88 failure regarding the use of infant photos in matching.

89 If adults have difficulty recognising infants, then this problem may persist when deciding

90 whether two photographs depict the same child. Furthermore, given that there are dramatic

91 changes in facial structure during childhood, comparing photographs of infants of similar ages

92 might even be considered an 'easy' context in relative terms. Many countries that require infants

93 to have their own passports allow them to be used for five years before expiration (e.g., Canada,

94 United Kingdom, United States, Australia). This means that officials may need to compare two

95 images (or an image and a live face) that differ in age by up to five years. Such age gaps likely

96 result in significant difficulties because infants' faces change substantially at a young age (e.g.,

97 Chakravarty et al., 2011; Farkas, Posnick, Hreczko, \& Pron, 1992; Ferrario, Sforza, Serrao,

98 Colombo, \& Ciusa, 1999), and unfamiliar face matching is closely bound to the visual properties

99 of the particular images (Hancock, Bruce, \& Burton, 2000). Consistent with this possibility, there

100 is evidence that hit rates (correct identification of targets) for adult faces decrease with only 17

101 months passing between two photographic sittings (Megreya, Sandford, \& Burton, 2013) and

102 that larger age gaps result in worse matching performance (using three images each of four

103 female students; Seamon, 1982). Indeed, with an adult face matching test featuring images with 
104 an average of only 9 months passing between sittings, performance was significantly lower than

105 for the equivalent test where images were taken only minutes apart (Fysh \& Bindemann, 2018).

106 Despite the importance of this issue for policy decisions, we were surprised to find only

107 two studies reporting on face matching with images of children, both of which support our

108 supposition that this task is particularly difficult. In a study on machine face recognition, Yadav,

109 Singh, Vatsa, and Noore (2014) found poor face matching performance for full-face images of

110 children (60\%, where chance level was 50\%) when the two photographs depicted an individual

111 once in the age range 0-5 years and again in the range 6-10 years. However, few details about the

112 human task were reported, and the two images presented did not differ by a constant age gap or

113 focus specifically on the change over the first five years of life. A similar conclusion was

114 reported by White, Dunn, Schmid, and Kemp (2015). In their task, for trials involving child

115 matching, the recent photograph depicted an individual aged between 6 and 13 years $(M=10.0$

116 years), while their previous photograph was taken an average of 6.2 years earlier. Overall

117 performance on these trials was 39\%, with accuracy on adolescent (41\%) and adult trials (45\%)

118 also very poor, and the latter resulting in statistically better performance in comparison with both

119 child and adolescent trials. Although age was not the focus of this work, these results highlight

120 just how difficult face matching can be with child images, and also provide evidence that this

121 task may be significantly more difficult than adult face matching.

122 Importantly, neither of these previous studies specifically examined face matching for

123 infants or focussed on the age range considered valid for passports. In the current work, we

124 investigate how difficult face matching is when the images are of infants and young children.

125 Our first experiment explored face matching when both images depicted infants ( $<1$ year old),

126 while our second experiment considered the five-year validity of child passports by pairing an 
127 infant's photograph with one of a child aged 4-5 years old. Experiments 1 and 2 examined

128 performance under optimistic conditions. In these experiments, there was no attempt to make it

129 difficult to detect matches and mismatches in the facial identities presented (e.g., identity pairs

130 were not systematically matched for sex, hair colour, etc.). In contrast, Experiment $3 a$ examined

131 performance when there was a deliberate attempt to make the identities look dissimilar on match

132 trials and similar on mismatch trials, while Experiment $3 \mathrm{~b}$ extended this further by specifically

133 including only same-sex mismatch pairings. Finally, Experiment 4 addressed this issue of

134 whether the sex of infants and children could be judged accurately from facial photographs, a

135 question which was raised by the first three experiments. Together, we aimed to investigate for

136 the first time how difficult these infant and child face matching tasks may be, and as a result, we

137 hoped to determine the utility of facial images in infant and child passports.

138

139 Experiment 1

140

141

This first experiment examined how accurate people were at deciding whether the infant

142 faces depicted in two photographs belonged to a single individual ('match' condition) or

143 different individuals ('mismatch' condition). Participants were shown pairs of images where both

144 photographs depicted infants in their first year of life and were asked to decide whether these

145 images showed the same infant or two different infants. Comparing two passport-style images

146 mirrors passport replacement and renewal procedures, which are typically carried out online or

147 by post and do not involve 'live matching' to a person. There was no attempt to pair the

148 identities in mismatch trials based upon visual similarity, permitting us to establish an upper-

149 bound for accuracy. 
150

\section{Method}

152

153 Participants

154

155 Thirty students ( 26 women; age $M=24.90$ years, $S D=9.68 ; 73.33 \%$ self-reported

156 ethnicity as White) at Trent University took part in exchange for course credits. All participants

157 in Experiments 1 and 2 provided written informed consent and were verbally debriefed at the end

158 of the experiment. Sample size was based on past research using a face matching paradigm

159 (Dowsett \& Burton, 2015; Estudillo \& Bindemann, 2014). Trent University's ethics committee

160 approved all experiments presented here (ref: 22305), which were carried out in accordance with

161 the provisions of the World Medical Association Declaration of Helsinki.

162

163 Stimuli

164

165 Images from the City Infant Faces Database (Webb, Ayers, \& Endress, 2018) were

166 obtained from its creators. These depicted 33 male and 35 female infants in a total of 154

167 photographs. In most cases, multiple images were available for each infant, with these typically

168 showing a negative, a neutral, and a positive expression (as the original goal of the database was

169 to investigate infant facial expressions).

170 Each parent was asked to provide multiple images of their infant, and was instructed to

171 take the photographs all at the same time of day and with the infant's head at the same angle in

172 each photograph. No record was kept of when the images were taken, and so these may all have 
173 been taken on the same day (at the minimum), or potentially with a few days or weeks between

174 them (at the maximum).

175 From this original set of 154 images, we considered only infants of White ethnicity where

176 two or more images were available. From this subset, we then excluded images with strong facial

177 expressions, eyes fully closed, strong lighting/shadows, or low resolution. These criteria were

178 used in order to comply with typical governmental guidelines regarding the appearance of

179 standard infant passport photographs. Often, however, guidelines are significantly more relaxed

180 than this (e.g., non-neutral expressions, indirect gaze, and closed eyes can be acceptable; HM

181 Passport Office, n.d.) since agencies acknowledge how difficult it can be to capture a controlled

182 infant facial photograph. Finally, where more than two images remained for a given infant, we

183 selected the two displaying expressions closest to neutral. Our final image set comprised 22 male

184 and 19 female infants (age range: 3-11 months), each with two different images that met

185 international standards for passport portraits.

186 All faces were cropped and shown in greyscale on a white background, and measured

187 approximately $5.5 \mathrm{~cm} \mathrm{x} 7 \mathrm{~cm}$ onscreen (see Fig. 1).

\section{Procedure}

190

191 The task comprised 41 match trials (different images of the same infant) and 41 mismatch

192 trials (images of two different infants). The former involved presenting both images of the infant 193 in the photoset (see above), while the latter were created by pairing one image of every infant

194 (chosen randomly from the two photos available) with an image of a different infant (again, 
195 chosen randomly). These infant pairings were created at random for each participant, resulting in 196 every identity appearing four times (two images in a match trial and two in mismatch trials). Importantly, and as discussed below, identities are not paired at random in real-world 198 contexts, where fraudulent passports would be selected in order to most resemble individuals. 199 Here, the random pairing of identities for mismatch trials meant that it was possible for faces to 200 differ in terms of hair colour, eye colour, and even sex. As such, the present study will likely 201 result in inflated estimates of accuracy.

202 Participants were tested individually in a computer laboratory. On each trial, two images 203 were presented onscreen, one to the left and one to the right of centre, using custom MATLAB 204 software. Viewing distance was not fixed. The task, which we explained verbally to participants 205 beforehand, was to judge whether the two images were of the same person or two different 206 people. Participants responded using the keyboard, pressing A for 'same' and L for 'different'.

207 These labels were presented at the top of the screen and remained visible throughout the 208 experiment. Trials were self-paced, and no feedback was given at any point during the experiment. The order of the trials was randomised, as was the location of each face (left or right 210 side) within each trial.

212 also asked if they had had regular contact with infants in the last few years. Unfortunately, very

213 few of our (university student) sample had such experience, and so we were unable to explore 214 this further in our analyses.

\section{Results}


For each participant, we calculated their overall percentage correct. In addition, following

219

220

221

222

223

224

225

226

227

228

229

230

231

232

233

234

235

236

237

238

239

240

other research in this field (e.g., Kramer \& Ritchie, 2016), we investigated signal detection

measures. We calculated sensitivity indices $\left(d^{\prime}\right)$ and criterion values $(c)$ using the following: Hit,

both images are of the same identity and participants responded 'same'; and False alarm, the two images are of different people and participants responded 'same'.

Our results are summarised in Table 1. For this experiment, we found that both percentage correct, $t(29)=19.98, p<.001$, Cohen's $d=3.65$, and $d$ ' sensitivity, $t(29)=16.44, p<.001$, Cohen's $d=3.00$, were significantly higher than chance levels. In addition, both hit rate, $t(29)=$ 9.83, $p<.001$, Cohen's $d=1.79$, and false alarm rate, $t(29)=9.93, p<.001$, Cohen's $d=1.81$,

were significantly better than chance levels. Finally, criterion was not significantly different from zero, $t(29)=0.48, p=.632$, Cohen's $d=0.09$, suggesting no bias in responses.

Table 1 also includes measures of performance for a benchmark test of face matching in order to provide some comparison regarding difficulty. Using the Glasgow face matching test (GFMT; Burton et al., 2010), researchers presented 168 pairs of passport-style photographs of adult faces and asked participants to decide whether the images were of the same person or two different people. The images were taken approximately 15 mins apart but with different cameras. Importantly, on mismatch trials, identities were paired on the basis of similarity, i.e., foil identities were those faces most similar to the target identities. This feature increases the difficulty of the task and therefore establishes the GFMT as a plausible estimate for adult matching accuracy (at least as applied to the specific identities featured). The present study was statistically compared (using $t$-tests with unpooled variances here and below for comparisons between studies) with the GFMT (long version, described here) using the means and standard deviation values reported by Burton and colleagues (2010). As Table 1 illustrates, accuracy on 
241 the GFMT was higher than our results presented here, $t(38)=15.19, p<.001$, Cohen's $d=2.49$.

242 The same pattern was also found for $d$ ' sensitivity, $t(55)=18.25, p<.001$, Cohen's $d=2.06$.

243 However, criterion was not significantly different in comparison with the GFMT, $t(35)=1.79, p$

$244=.082$, Cohen's $d=0.34$.

245 As noted earlier, there was no attempt to pair infants in mismatch trials based on

246 appearance. Indeed, our random pairing of identities on mismatch trials meant that faces with

247 different hair and eye colour were compared, and even male and female faces. As such, the poor

248 performance levels with infant matching reported here may represent an upper estimate in

249 applied situations (e.g., border control), in that pairing identities based upon visual similarity

250 would increase the difficulty of mismatch trials (see Experiment 3). At best, this suggests that

251 border control officers would incorrectly identify $27 \%$ (95\% CI [23\%, 32\%]) of the 400,000

252 infant cases mentioned earlier if they were all presented in 'mismatch' contexts. In order to gain

253 some insight into the extent to which we were overestimating accuracy levels, we elected to

254 compare percentage correct on mismatch trials where the two identities were the same versus

255 different with respect to sex. Interestingly, we found no difference in performance, $t(29)=0.81$,

$256 p=.425$, Cohen's $d=0.12$. (Although a within-participants comparison, we report Cohen's $d$

257 using the pooled estimate of the standard deviation as the standardiser here and throughout, more

258 easily allowing for comparisons with other studies irrespective of their designs.) This is

259 surprising, given that research has shown that adult participants were able to categorise the sex of

260 neonates (Kaminski, Méary, Mermillod, \& Gentaz, 2011; Porter, Cernoch, \& Balogh, 1984;

261 Round \& Deheragoda, 2002) and 1-24 month old infants (Tskhay \& Rule, 2016) at levels above

262 chance. Indeed, the sex of infant faces may be perceived automatically (Tskhay \& Rule, 2016).

263 However, as mentioned earlier, sex characteristics are more evident after puberty and are likely 
264 subtle where present in infants. Therefore, either participants were unable to categorise our infant

265 faces by sex or they neglected to use this information when making same/different judgements.

266 We return to this issue in Experiments 3 and 4.

267

268 Experiment 2

Although Experiment 1 established that matching with infant faces is more difficult than

270 with adult faces, our task was limited to images taken with minimal time passing between

271 photographic sittings. In reality, professionals are required to carry out face matching

272 comparisons with images that were taken up to five years beforehand. Experiment 2 therefore 273 examined face matching performance for photos taken approximately 4 to 5 years apart. Each

274 image pair showed an infant (less than one year old) paired with a child aged 4-5 years old.

275 Again, the task was to determine whether the two images showed the same individual (match

276 trials) or different individuals (mismatch trials). It was expected that significant aging across

277 images would have detrimental effects on performance. As with Experiment 1, we made no

278 attempt to pair identities in mismatch trials based upon visual similarity. This should make

279 discrimination of match and mismatch trials relatively easy, and permits direct comparison with

280 Experiment 1 . As we were unaware of any database containing photos of infants across the age

281 range 0-5 years, we elected to use photos of celebrities' children due to their widespread

282 availability online. This approach resulted in less control over the photographs with regard to

283 pose and facial expression. The use of child images that incorporate more variation than typical

284 passport photos meant that this task was comparable to a border control situation, where a 'live'

285 face is matched to an infant passport image. Similarly, evidence collected in child abuse cases

286 often features more unconstrained images than passports allow. 
288 Method

289

290 Participants

291

292

Thirty students (26 women; age $M=25.00$ years, $S D=8.09 ; 76.67 \%$ self-reported

293 ethnicity as White) at Trent University took part in exchange for course credits. There was no

294 overlap between this sample and those who participated in Experiment 1. Sample size was again

295 based on past research using a face matching paradigm (Dowsett \& Burton, 2015; Estudillo \&

296 Bindemann, 2014).

297

298

Stimuli

299

300

Images were downloaded from the Internet using Google Image searches for the names of

301 celebrities' children (e.g., Suri Cruise, the daughter of Tom Cruise and Katie Holmes). We chose

302 to collect photographs from this population because images were often available for the same

303 child at different ages due to extensive media coverage. Using the child's birthdate and the

304 earliest dates when images were posted online, we were able to calculate the approximate age of 305 the child for each image.

306 For each of 30 children (White ethnicity; 15 female), we collected two photographs - one

307 as an infant (age range: 3 months to 1 year old) and the other as a child (age range: 4 to 5 years

308 old, with the exception of one 6-year-old). Infant images were selected to comply with typical

309 governmental guidelines regarding the appearance of standard infant passport photographs (see 
310 Experiment 1). The photographs of the children were taken approximately front-on, with the

311 majority looking directly into the camera and posing with a relatively neutral expression.

312 However, due to the difficulties inherent in collecting these types of images of children, we also

313 accepted slight head turns, as well as some facial expressions (typically, a smile). We felt that

314 some minor deviations from passport photograph guidelines were acceptable for this task and

315 mirrored real-world appearances that might be presented in border control contexts. For this

316 reason, we also chose to leave the child photos uncropped (see Fig. 2). All faces were rotated so

317 that both pupils were aligned to the same transverse plane, and were shown in greyscale on a

318 white background. Images measured approximately $5.5 \mathrm{~cm} \mathrm{x} 7 \mathrm{~cm}$ onscreen.

\section{Procedure}

321

The procedure was identical to the one used in Experiment 1, except for the use of different 323 stimuli. Here, 30 match trials and 30 mismatch trials were presented. The former involved

324 presenting both images of the identity (one infant photo and one child photo), while the latter

325 were created by pairing every identity's infant photo with a different identity's child photo

326 (chosen randomly for each participant). In this way, every image appeared twice during the task,

327 once in a match trial and once in a mismatch trial. Participants were instructed onscreen at the 328 start of the experiment that they would be shown photographs of an infant (1 year old or less) 329 and a child (aged 4-5 years), and that their task was to judge whether the two images were of the 330 same child or not.

331 Given that our images depicted celebrities' children, we asked participants upon

332 completion of the task whether they had recognised any of the identities in the experiment. One 
333 participant responded that they had only recognised one identity. We therefore decided not to 334 exclude any data from the subsequent analyses.

335 Finally, demographic information was collected, and participants were additionally asked

336 if they had had regular contact with infants in the last few years. Unfortunately, as in Experiment

337 1, very few of our (university student) sample had such experience, and so we were unable to 338 explore this further in our analyses.

339

340 Results

341

342

The same performance indicators were examined as in Experiment 1, and can be seen in

343 Table 1. We found that both percentage correct, $t(29)=10.22, p<.001$, Cohen's $d=1.87$, and $d$ '

344 sensitivity, $t(29)=9.46, p<.001$, Cohen's $d=1.73$, were significantly higher than chance levels.

345 In addition, both hit rate, $t(29)=6.67, p<.001$, Cohen's $d=1.22$, and false alarm rate, $t(29)=$

$3467.73, p<.001$, Cohen's $d=1.41$, were significantly better than chance levels. Finally, criterion

347 was not significantly different from zero, $t(29)=0.58, p=.567$, Cohen's $d=0.11$, suggesting no

348 bias in responses. For this experiment, we found performance levels that were significantly lower

349 than for Experiment 1: percentage correct, $t(54)=4.25, p<.001$, Cohen's $d=1.10$, and $d$ '

350 sensitivity, $t(57)=4.27, p<.001$, Cohen's $d=1.10$. However, we found no difference between

351 the two experiments with regard to criterion, $t(50)=0.13, p=.897$, Cohen's $d=0.03$.

352 As with Experiment 1, performance in this study was statistically compared with the long 353 version of the GFMT. As Table 1 illustrates, performance on the GFMT was higher than our 354 percentage correct presented here, $t(34)=17.36, p<.001$, Cohen's $d=3.48$. The same pattern 
355 was also found for $d$ ' sensitivity, $t(52)=22.39, p<.001$, Cohen's $d=2.65$. In addition, criterion

356 was significantly lower for the GFMT, $t(44)=2.36, p=.023$, Cohen's $d=0.32$.

357 Our performance level here (64\%) was similar to the low levels of accuracy found in

358 previous research investigating matching across age gaps where individuals were depicted once

359 in the age range 0-5 years and again in the range 6-10 years (Yadav et al., 2014). Taken together,

360 it seems clear that comparing an infant's photograph to a real-world image (a little more

361 variation than a typical passport photo) is a highly difficult task, suggesting that border control

362 officers are faced with a significantly error-prone situation.

363 As in Experiment 1, we compared accuracy on mismatch trials where the two identities

364 were the same versus different with respect to sex. For each participant, we calculated their

365 percentage correct on these two trial types, with a paired samples $t$-test showing no difference in 366 performance, $t(29)=0.69, p=.496$, Cohen's $d=0.17$. Again, we found no evidence that

367 participants were able to categorise our faces by sex and/or use this information when matching.

368 Most likely, this was a failing with the infant faces (see Experiment 1) since the child

369 photographs (aged 4-5 years) included both hair and some clothing information that made sex

370 categorisation fairly easy.

371

372 Experiment 3a

373

374

This experiment had three objectives. First, we wanted to examine performance under

375 more difficult conditions. In order to make our tasks harder, we followed the same procedure used by Burton and colleagues (2010) when creating the short version of the GFMT. Difficult versions of our two tasks were created by selecting those trials which demonstrated lowest 
378 accuracy in previous participants. By selecting the most difficult trials in our infant and child 379 matching tasks, we mimic realistic cases of fraud where identities are typically selected to be the 380 most likely to fool the authorities. Second, we wanted to compare performance directly with the 381 short version GFMT (Burton et al., 2010), which provides a more difficult test of adult face 382 matching. Third, by asking each participant to match faces under all three conditions (infant 383 only, infant-child, and adult only using the GFMT), we were able to directly compare 384 performance across stimuli, but also to correlate performance measures in order to determine 385 whether an individual's matching ability in one task was predictive of scores on the other two 386 tasks. Previous research has shown that individuals who perform well on one measure of face 387 matching are typically good at other matching tasks (Bobak, Dowsett, \& Bate, 2016).

388

389

390

391

392

393

394 395 396 397 398 399 400

Participants

\section{Method}

$$
\text { A community sample of } 114 \text { participants ( } 49 \text { women; age } M=32.86 \text { years, } S D=9.77 \text {; }
$$

65.79\% self-reported ethnicity as White) were obtained via Amazon's Mechanical Turk (MTurk) in exchange for $\$ 1.50$ in payment. There was no overlap between this sample and those who participated in earlier experiments. All participants provided informed consent online and were shown a debriefing screen at the end of the experiment. All participants were unique (due to the nature of the project specifications on MTurk) and so no data were excluded because of repeated participation by the same individuals. 


\section{Stimuli}

402

403 Images from the short version of the GFMT (Burton et al., 2010) were used to assess

404 performance for adult face matching under difficult conditions. The task comprised forty pairs of

405 adult male (24) and female faces (16) viewed front on, where half the pairs were match trials

406 (different images of the same person) and half were mismatch trials (different people with a

407 similar appearance). The 40 face pairings were taken from the original GFMT set of 168 pairs

408 (described above) and represent the most difficult trials (based on the performance of 300

409 participants).

410 We took similar steps to construct more difficult versions of our two infant matching tasks.

411 In order to select the most difficult 20 match and 20 mismatch trials in each case, we analysed

412 the 'by trial' accuracies for Experiments 1 and 2, and chose the identity pairings that resulted in

413 the lowest performance. This approach was reasonable for match trials because there were 30

414 observations for each image pair. However, this was more problematic for mismatch pairs

415 because identities were paired randomly in these experiments. As such, specific pairings

416 occurred infrequently and accuracies were therefore based on small numbers of observations. In

417 all cases, we selected only mismatch trials where the mean accuracy was $0 \%$ (i.e., no participant

418 made the correct response), although in some cases, trials were only encountered once

419 previously. As a result, our difficulty manipulation may not have been as powerful as the one

420 used by Burton et al. (2010). As with the short version of the GFMT, we made no attempt to

421 prevent identities/images appearing more than once (e.g., a particular infant may resemble

422 several others, resulting in their presentation in multiple difficult mismatch trials). As before, we

423 did not restrict ourselves to same-sex pairings in mismatch trials. 
424 All faces were shown in greyscale and measured approximately $6 \mathrm{~cm} \mathrm{x} 8 \mathrm{~cm}$ onscreen.

425

426

427

428

429

430

431

432

433

434

435

436

437

438

439

440

441

442

443 inserted into the GFMT short version's trial order for each participant. For one trial, a female

444

445

\section{Procedure}

The experiment was completed online through the Testable website (www.testable.org).

First, participants were instructed to set their browsing windows to full screen, minimise possible distractions (e.g., TV, phone, etc.), and position themselves at arm's length from the monitor for the duration of the experiment (although viewing distance was not fixed). Next, a screen size calibration took place (adjusting an onscreen bar to match the length of a credit card), consent was obtained, and then demographic information was collected.

On each of the 120 trials ( 3 tasks x 40 trials), two images were presented onscreen, one to the left and one to the right of centre. The task was to judge whether the two images were of the same person or two different people. Participants responded using the keyboard, pressing A for 'same' and L for 'different'. These labels remained onscreen throughout the experiment. Trials were self-paced, and no feedback was given at any point during the experiment. The trials were blocked by task (infant only, infant-child, and adult only), with the trial order randomised within each task. The task order was also randomised for each participant.

In order to check whether participants were concentrating during the experiment (since this can be a concern for online studies), we included two additional trials that were randomly image from one of the test trials was paired with itself. Because these were two identical images, participants were expected to respond 'same'. For the other trial, a male image from one of the 
446 test trials was paired with a (different) female test image. Because these images depicted a man

447 and a woman, participants were expected to respond 'different'.

448

449 Results

450

451

The same performance indicators were examined as in Experiments 1 and 2, and can be

452

seen in Table 1. Data from 21 participants were excluded because they responded incorrectly to one or both of the 'checking' trials. Percentage correct scores for the remaining 93 participants' data were analysed using a repeated measures analysis of variance (ANOVA), comparing the three tasks. We found a significant effect of task, $F(2,184)=165.10, p<.001, \eta_{\mathrm{p}}^{2}=.64$, with pairwise comparisons (Dunn-Šidák corrected here and below) revealing that participants $p$ s $<.001)$. In addition, percentage correct scores were significantly lower in the 'infant-child'

459

460

461

462

463

464

465

466

467 468 task in comparison with the 'infant-infant' task $(p=.003)$.

We also carried out analyses at a more fine-grained scale, considering percentage correct separately on match and mismatch trials. A 3 (Task: infant only, infant-child, adult only) x 2 (Trial Type: match, mismatch) within-subjects ANOVA found a significant main effect of Task, $F(2,184)=165.10, p<.001, \eta_{\mathrm{p}}^{2}=.64$, but no main effect of Trial Type, $F(1,92)=1.90, p=$ $.171, \eta_{\mathrm{p}}^{2}=.02$. However, these effects were qualified by a significant Task $\mathrm{x}$ Trial Type interaction, $F(2,184)=34.94, p<.001, \eta^{2}{ }_{\mathrm{p}}=.28$. We therefore considered the simple main effects of Task at each level of Trial Type. These simple main effects were significant for both match, $F(2,184)=120.34, p<.001, \eta_{\mathrm{p}}^{2}=.57$, and mismatch trials, $F(2,184)=47.06, p<.001$, $\eta_{\mathrm{p}}^{2}=.34$. Pairwise comparisons showed that, for match trials, accuracies on all three tasks 
469 significantly differed from each other (all $p \mathrm{~s}<.001$ ), in descending order of adult only, infant-

470 child, then infant only. For mismatch trials, accuracies on all three tasks also significantly

471 differed from each other (all $p$ s $<.051$ ), in descending order of adult only, infant only, then

472 infant-child.

473 An analysis of the $d$ ' sensitivities for the three tasks showed the same pattern of results as

474 for percentage correct scores (see Table 1). We found a significant effect of task, $F(2,184)=$

$475165.87, p<.001, \eta_{\mathrm{p}}^{2}=.64$, with pairwise comparisons revealing that participants showed higher

476 sensitivity on the short version of the GFMT in comparison with the other two tasks (both $p$ s $<$

$477.001)$. In addition, $d$ ' sensitivities were significantly lower in the 'infant-child' task in

478 comparison with the 'infant-infant' task $(p=.001)$.

479 An analysis of criterion found a significant effect of task, $F(2,184)=31.86, p<.001, \eta^{2} \mathrm{p}=$

480 .26. Pairwise comparisons revealed that criterion was significantly higher for the 'infant-infant'

481 task in comparison with the other two tasks (both $p \mathrm{~s}<.001$; see Table 1), with these two tasks

482 not differing from each other $(p=.560)$.

483 Although performance was very low in the two infant tasks, we did find that both

484 percentage correct (both $p \mathrm{~s}<.002$ ) and $d$ ' sensitivity (both $p \mathrm{~s}<.003$ ) remained significantly

485 higher than chance levels on each task. Criterion did not differ from zero in the 'infant-child'

486 task, $t(92)=1.10, p=.273$, Cohen's $d=0.11$, suggesting no bias in responses. However, this

487 measure was significantly above zero in the 'infant-infant' task, $t(92)=5.97, p<.001$, Cohen's $d$

$488=0.62$, suggesting a bias towards responding 'different'.

489 As in Experiments 1 and 2, we compared accuracy on mismatch trials where the two

490 identities were the same versus different with respect to sex. For each participant, we calculated

491 their percentage accuracy on these two trial types, separately for the two infant tasks. Paired 
492 samples $t$-tests showed no difference in performance for the 'infant-child' task, $t(92)=1.10, p=$ 493.275 , Cohen's $d=0.11$, but a significant difference for the 'infant-infant' task, $t(92)=4.47, p<$ 494.001 , Cohen's $d=0.46$. This result suggests that, in contrast with Experiment 1, participants' 495 accuracies were higher on mismatch trials where a male and a female infant were presented 496 together $(M=68.9 \%)$ in comparison with two same-sex infants $(M=59.6 \%)$ for this image set.

497 These findings provide mixed support for previous research where infant sex was shown to be 498 accurately judged from facial images (e.g., Tskhay \& Rule, 2016).

499 In contrast with Experiments 1 and 2, the identity pairings, and hence the trials, for our two 500 infant tasks here were the same for all participants. This allowed us to measure the internal 501 reliability of the two tasks. For each task, we randomly divided the 20 match trials into two sets 502 of ten. We then calculated participants' accuracies for these two sets of trials separately (always 503 using the same two sets, irrespective of the actual order in which they were presented during the 504 task). As a measure of split-half reliability, we correlated these two accuracies across 505 participants, finding significant associations for both the 'infant-infant' task, $r(91)=.36, p<$ 506.001 , and 'infant-child' task, $r(91)=.31, p=.002$. Carrying out the same process for mismatch 507 trials, we again found significant associations for both the 'infant-infant' task, $r(91)=.56, p<$ 508.001 , and 'infant-child' task, $r(91)=.41, p<.001$. These values were likely lower than for 509 previous tests (e.g., $r=.81$ for the GFMT; Burton et al., 2010) due to the low number of trials in 510 each 'half'. However, it was important to consider the match and mismatch trials separately

511 because previous research has found a dissociation between accuracies on these two trial types 512 (Megreya \& Burton, 2007).

513 Finally, we investigated within-person performance across all three tasks. It is well

514 established that face matching ability appears to be a stable trait that generalises across different 
515 tasks (e.g., Bobak, Dowsett, et al., 2016; Robertson, Noyes, Dowsett, Jenkins, \& Burton, 2016),

516 as well as different versions of the same task (e.g., frontal and profile versions of the GFMT;

517 Kramer \& Reynolds, 2018). For instance, Bobak, Dowsett, and colleagues (2016) reported a

518 correlation of .72 between participants' $d$ 'values on the GFMT and a second face matching task.

519 Here, we found that $d$ ' sensitivities for the short version of the GFMT showed medium-sized

520 correlations with both the 'infant-infant' task, $r(91)=.35, p=.001$, and 'infant-child' task, $r(91)$

$521=.40, p<.001$. A similar-sized association was also found between the two infant tasks, $r(91)=$

$522.28, p=.007$. Although not as large as the correlation between adult matching tasks previously

523 reported, these results may suggest that an underlying ability with faces supports both adult and

524 infant matching performance. However, it is important to note that $d$ ' values were very low and

525 close to chance levels for both infant tasks. With such poor performance, any apparent

526 associations between tasks may simply be due to noise. Therefore, the next experiment will

527 determine whether these within-person correlations can be replicated.

528

529 Experiment 3b

530

531 The results of Experiment 3a suggested that, in contrast with Experiment 1, participants

532 were able to use information regarding the sex of infants in order to perform more accurately on

533 mismatch trials where a male and a female infant were presented together. We therefore decided

534 to rerun Experiment 3a while presenting only same-sex mismatch trials. This would provide us

535 with a full replication of the main result (adult face matching is easier than infant matching tasks)

536 while simulating more real-world contexts, where fraudulent passports would be selected in

537 order to most resemble individuals. If sex information is available in infant facial photographs 
538 then identity pairings would certainly be matched by fraudsters on this dimension. Finally, this

539 experiment will allow us to determine how robust the within-person performance correlations are

540 that were revealed by Experiment 3a.

541

542 Method

543

544 Participants

545

546 A community sample of 130 participants (53 women; age $M=33.18$ years, $S D=9.79$;

$54747.69 \%$ self-reported ethnicity as White) were obtained via MTurk in exchange for $\$ 1.50$ in

548 payment. There was no overlap between this sample and those who participated in earlier

549 experiments. All participants provided informed consent online and were shown a debriefing

550 screen at the end of the experiment. All participants were unique (due to the nature of the project

551 specifications on MTurk) and so no data were excluded because of repeated participation by the 552 same individuals.

553

554 Stimuli

555

556 The stimuli used here were identical to those used in Experiment 3a. However, when

557 selecting difficult mismatch trials for the 'infant-infant' and 'infant-child' tasks, we only

558 included same-sex identity pairings.

559

560 Procedure 
561

562

This was identical to the procedure used in Experiment $3 a$.

563

564 Results

565

566

The same performance indicators were examined as in Experiments 1 and 2, and can be

567

seen in Table 1. Data from 11 participants were excluded because they responded incorrectly to

568

one or both of the 'checking' trials. Percentage correct scores for the remaining 119 participants'

569 data were analysed using a repeated measures ANOVA, comparing the three tasks. We found a

570 significant effect of task, $F(2,236)=157.17, p<.001, \eta_{\mathrm{p}}^{2}=.57$, with pairwise comparisons

571 revealing that participants performed better on the short version of the GFMT in comparison

572 with the other two tasks (both $p$ s $<.001$ ). In addition, percentage correct scores were

573 significantly lower in the 'infant-child' task in comparison with the 'infant-infant' task $(p<$

$574.001)$.

575 We also carried out analyses at a more fine-grained scale, considering percentage correct

576 separately on match and mismatch trials. A 3 (Task: infant only, infant-child, adult only) x 2

577 (Trial Type: match, mismatch) within-subjects ANOVA found a significant main effect of Task,

$578 F(2,236)=157.17, p<.001, \eta_{\mathrm{p}}^{2}=.57$, and of Trial Type, $F(1,118)=6.20, p=.014, \eta_{\mathrm{p}}^{2}=.05$.

579 However, these effects were qualified by a significant Task $\mathrm{x}$ Trial Type interaction, $F(2,236)=$

$58027.57, p<.001, \eta_{\mathrm{p}}^{2}=.19$. We therefore considered the simple main effects of Task at each level

581 of Trial Type. These simple main effects were significant for both match, $F(2,236)=82.42, p<$

$582.001, \eta_{\mathrm{p}}^{2}=.41$, and mismatch trials, $F(2,236)=42.98, p<.001, \eta_{\mathrm{p}}^{2}=.27$. Pairwise comparisons

583 showed that, for match trials, accuracies on all three tasks significantly differed from each other 
584 (all $p \mathrm{~s}<.001$ ), in descending order of adult only, infant-child, then infant only. For mismatch

585 trials, accuracies were lower for the infant-child task in comparison with the other two tasks

586 (both $p s<.001)$. However, the adult only and infant only tasks did not differ $(p=.520)$.

587 An analysis of the $d$ ' sensitivities for the three tasks showed the same pattern of results as

588 for percentage correct scores (see Table 1). We found a significant effect of task, $F(2,236)=$

$589169.54, p<.001, \eta_{\mathrm{p}}^{2}=.59$, with pairwise comparisons revealing that participants showed higher

590 sensitivity on the short version of the GFMT in comparison with the other two tasks (both $p$ s $<$

591 .001). In addition, $d$ ' sensitivities were significantly lower in the 'infant-child' task in

592 comparison with the 'infant-infant' task $(p<.001)$.

593 An analysis of criterion found a significant effect of task, $F(2,236)=24.78, p<.001, \eta^{2} \mathrm{p}=$

594 .17. Pairwise comparisons revealed that criterion was significantly higher for the 'infant-infant'

595 task in comparison with the other two tasks (both $p$ s $<.001$; see Table 1), with these two tasks

596 not differing from each other $(p=.996)$.

597 Although performance was very low in the two infant tasks, we did find that both

598 percentage correct (both $p \mathrm{~s}<.030$ ) and $d$ ' sensitivity (both $p$ s $<.037$ ) remained significantly

599 higher than chance on each task. Criterion did not differ from zero in the 'infant-child' task,

$600 t(118)=0.44, p=.658$, Cohen's $d=0.04$, suggesting no bias in responses. However, this

601 measure was significantly above zero in the 'infant-infant' task, $t(118)=6.33, p<.001$, Cohen's

$602 d=0.58$, suggesting a bias towards responding 'different'.

603 Finally, we investigated within-person performance across all three tasks. Here, we found

604 that $d$ ' sensitivities for the short version of the GFMT and the 'infant-child' task showed a

605 medium-sized correlation, $r(117)=.26, p=.004$. However, there was no association between the

606 two infant tasks, $r(117)=.14, p=.143$, or the 'infant-infant' task and the GFMT, $r(117)=.17, p$ 
$607=.067$. These results cast doubt on the correlational findings of Experiment 3a, suggesting that 608 any underlying face matching ability may be weaker than was found earlier. As discussed above, 609 the low level of performance for the infant tasks, and the possibility of a floor effect, provide a 610 restricted range with which to investigate any associations, with the risk that any apparent 611 relationships may simply be the result of noise. As such, further work designed specifically to 612 address this issue is needed before firm conclusions can be drawn.

613

\section{Experiment 4}

615

616

Experiments 1 and 2 provided no evidence that participants were able to perceive or utilise

617 sex information in order to increase performance on mismatch trials where a male and a female 618 identity were presented together. However, the results of Experiment 3a suggested that, at least

619 for 'infant-infant' trials, sex information was indeed beneficial when making same/different 620 judgements. In this final experiment, we therefore decided to investigate whether participants 621 could perceive the sex of infants and children from facial photographs.

622

623 Method

624

625

Participants

626

627 A community sample of 40 participants (16 women; age $M=32.20$ years, $S D=9.36$;

$62870.00 \%$ self-reported ethnicity as White) were obtained via MTurk in exchange for $\$ 1.50$ in 629 payment. There was no overlap between this sample and those who participated in earlier 
630 experiments. All participants provided informed consent online and were shown a debriefing

631 screen at the end of the experiment. All participants were unique (due to the nature of the project

632 specifications on MTurk) and so no data were excluded because of repeated participation by the

633 same individuals.

634

635 Stimuli

636

637

The stimuli used here were identical to those featured in Experiments 1 and 2. Specifically,

638 we included two images each for 41 identities (Experiment 1) and 30 identities (Experiment 2).

639 This set of 142 images comprised 82 infant faces (Experiment 1), 30 infant faces (Experiment 2), 640 and 30 child faces (Experiment 2).

641

642 Procedure

643

644 As with Experiments $3 a$ and $3 b$, this experiment was completed online through the

645 Testable website. Identical calibration, consent, and debriefing procedures were also used here.

646 On each of the 142 trials, a single image was presented centrally onscreen. The task was to

647 judge whether the image depicted a boy or a girl. Participants responded using the keyboard,

648 pressing $\mathrm{M}$ for 'male' and $\mathrm{F}$ for 'female'. These labels remained onscreen throughout the

649 experiment. Trials were self-paced, and no feedback was given at any point during the

650 experiment. The trial order was randomised for each participant and was not blocked by task.

651

652 Results 
653

654

No data were excluded because the minimum percentage correct score for the children's

655

images (aged 4-5 years old and hence expected to be easier to judge accurately) was $67 \%$. We

656 therefore had no reason to believe that any participants were not paying attention during the

657 experiment.

658 Percentage correct scores were analysed using a repeated measures ANOVA, comparing

659 the three sets of images (Experiment 1 infants, Experiment 2 infants, Experiment 2 children). We

660 found a significant effect of image set, $F(2,78)=271.70, p<.001, \eta_{\mathrm{p}}^{2}=.87$, with pairwise

661 comparisons revealing that participants performed differently across the three sets (all $p s<.001$ ).

662 Accuracy was highest for the Experiment 2 children $(M=82.3 \%, S D=6.6 \%)$, followed by the

663 Experiment 1 infants $(M=56.7 \%, S D=5.5 \%)$, and then finally the Experiment 2 infants $(M=$

$66450.7 \%, S D=7.1 \%)$.

665 We found that performance was significantly higher than chance levels for both

666 Experiment 1 infants, $t(39)=7.74, p<.001$, Cohen's $d=1.22$, and Experiment 2 children, $t(39)$

$667=30.72, p<.001$, Cohen's $d=4.86$, suggesting that sex information was present in these image

668 sets. However, participants' accuracies were no different from chance for the Experiment 2

669 infants, $t(39)=0.60, p=.555$, Cohen's $d=0.09$. These results align with the findings of

670 Experiment 3a, where performance was only higher for different-sex in comparison with same-

671 sex mismatch trials for Experiment 1 images. Taken together, we can conclude only that limited

672 information (accuracy was 57\% for Experiment 1 images) regarding infant sex is present in some

673 cases but not others, suggesting the need for further work in this area.

674

\section{General Discussion}


676

677

Our results provide compelling evidence that matching two images of infants was difficult

678 (72\%; Experiment 1$)$, and significantly more so than with two images of adult faces taken from a 679 university population (around 87-90\%; Bobak, Dowsett, et al., 2016; Burton et al., 2010). As we 680 might expect, task performance was significantly lower still when we introduced a five-year age 681 gap between the two images (64\%; Experiment 2). Importantly, our estimates of accuracy in 682 these two experiments may even be higher than those found in similar real-world contexts since 683 characteristics such as sex, hair colour, etc., differed on some mismatch trials. For those who 684 make use of fraudulent passports (altered in some way, or simply not their own), the choice of 685 who should be paired with which document/photograph will be driven by facial similarity, which 686 makes the job of spotting mismatches that much harder.

687 Experiments $3 \mathrm{a}$ and $3 \mathrm{~b}$ addressed how performance changes under more challenging

688 discrimination conditions by examining decisions when presented with more difficult versions of 689 our two tasks. Only the lowest performance trials were included in order to simulate purposeful 690 (rather than random) pairing of infants, as we would predict in real-world fraudulent documents.

691 In addition, Experiment $3 \mathrm{~b}$ included only same-sex pairings on mismatch trials. Unsurprisingly, 692 performance levels in these experiments were closer to chance (52-56\%). This clearly 693 demonstrated that, at its most difficult, using face photographs of infants provided almost no 694 useful information, with accuracies significantly lower than those found with adult faces 695 comparatively selected for difficulty. Importantly, even recent adult matching tasks, specifically 696 constructed to be challenging, showed higher levels of accuracy $-66 \%$ for the short version of 697 the Kent face matching test (Fysh \& Bindemann, 2018), 72\% for matching with male models 698 (Dowsett \& Burton, 2015), and 83\% for other-race faces (Kokje, Bindemann, \& Megreya, 2018). 
We constructed our more difficult task versions through selecting those trials which

700

701

702

703

704

705

706

707

708

709

710

711

712

713

714

715

716

717

718

719

720

721

demonstrated low accuracy in previous participants (mirroring construction of the short version

of the GFMT; Burton et al., 2010). While this does not tell us why these particular trials were

difficult, we can provide some initial insights through inspection of the image pairings used in

Experiments $3 \mathrm{a}$ and $3 \mathrm{~b}$. First, match trials resulted in poor performance when the image

characteristics (e.g., lighting direction, image quality, facial expression) significantly differed

across the two photographs. For the 'infant-child' task, changes in hair colour or style were often

present, suggesting that participants found it hard to ignore these details even though such

differences could be expected for an infant over a five-year period. Second, and as a

consequence, difficult mismatch trials presented two images where these superficial

characteristics were similar. Previous research has demonstrated that unfamiliar matching relies

heavily on the visual properties of the particular images (Hancock et al., 2000), and evidence

suggests an increasing reliance on the internal facial features (eyes, nose, and mouth) as we

become more familiar with a face (e.g., Clutterbuck \& Johnson, 2002, 2004, 2005), given that

the external features (hair, facial outline, etc.) contain less identity information (Kramer, Manesi,

Towler, Reynolds, \& Burton, 2018). Taken together, it is no surprise that image characteristics

and external features strongly influenced matching decisions here.

If infant matching suffers from an over-reliance on the external facial features, it may be

possible to improve performance on this task through instructing participants to ignore these

potentially uninformative sources of information. Indeed, there is some evidence to suggest that

performance with matching unfamiliar adult faces can be improved by displaying only the

internal features (Kemp, Caon, Howard, \& Brooks, 2016). However, this advantage was limited

to the most difficult trials only and failed to generalise to a card sorting task (Kramer et al., 
722 2018). Further research might consider drawing attention towards or away from certain features

723 of the face in order to improve infant matching performance.

724 While we might consider various methods that could result in performance increases, it

725 may be that even the highest performing humans and machine algorithms will eventually hit a

726 relatively low maximum level. This is because children's faces appear to be more homogeneous

727 than adult faces, displaying lower levels of between-face variability. With less information to

728 distinguish between identities, such images could simply be insufficient for useful identification

729 and matching in real-world situations. Indeed, performance in the current work certainly suggests

730 that any effective method of improving matching will continue to fall short of practical

731 requirements for what is acceptable in terms of accuracy.

732 We found a substantial drop in performance when pairs of images depicted a five-year age

733 gap (Experiment 2). Research with adult face matching has shown that images taken only

734 minutes apart produced significant levels of error (Burton et al., 2010), with performance

735 decreasing even further as months passed by between photographic sittings (Fysh \& Bindemann,

736 2018; Megreya et al., 2013). Here, we investigated a larger time frame and an infant sample, a

737 combination which was particularly likely to exhibit sizable appearance changes. As such,

738 although important to demonstrate, that matching under these conditions was difficult for our

739 participants came as no surprise. Whether infant face images provide sufficient information for

740 effective identification in real-world scenarios has yet to be determined.

741 When attempting to compare performance across different types of stimuli, it is important

742 to consider practically how this can best be achieved. There are two separate, yet related issues.

743 One concerns how the comparisons inform the situations that are likely to be encountered in our

744 everyday lives and the second concerns the inferences that can be made about the fundamental 
745 cognitive processes that underlie processing of the stimuli. With regards to the former, here we

746 have demonstrated that, on the whole, it is more difficult to match identities using infant faces

747 than adult faces. However, with regard to the latter, we cannot address whether the same

748 cognitive processes are used when matching identities with infant faces and adult faces. If this

749 were the case, then the performance differences could be explained in terms of the

750 presence/absence of information in the stimuli (e.g., infants were harder to match than adults

751 because sex characteristics were less salient). If infant and adult face matching utilise different

752 cognitive processes (e.g., does emotion play a role in infant face matching?) then they will

753 constitute different tasks and may yield different performance levels, even when the same

754 information is present. One approach might be to construct both types of stimuli using the same

755 identities and pairings but this would require images of each unfamiliar person as an infant,

756 child, and adult, which is logistically problematic. Perhaps more achievable, researchers might

757 consider performance with adult, infant, and child faces that systematically very on a set of

758 characteristics so that the contribution of each feature can be assessed across stimulus types.

759 Future research should consider how to further address this complex issue.

760 In Experiments 1 and 2, we found that participants were no better on mismatch trials when

761 the two identities were of different sexes. This result appears to contradict previous work

762 showing that infant and neonate faces could be categorised accurately by sex (Kaminski et al.,

763 2011; Tskhay \& Rule, 2016). However, in these studies, accuracy levels were always low,

764 despite being statistically above chance. The results of Experiments $3 \mathrm{a}$ and 4 provided additional

765 evidence more in line with past research, suggesting that sex information is present in some

766 image sets, although even in those cases, performance remained close to chance levels.

767 Therefore, participants in the current work may have benefitted to some degree on different-sex 
768 trials, but this advantage failed to produce any noticeable gain in accuracy (see Experiments 3a

769 versus $3 b$ in Table 1), perhaps because other, more salient features may have driven judgements.

770 We found some evidence of within-person correlations in performance across our two

771 infant tasks and the short version of the GFMT. However, these small to medium effects

772 (Experiment 3a) were unlike the large correlations found in previous work when researchers

773 considered two tasks of adult face matching (Bobak Dowsett, et al., 2016). Indeed, these

774 associations either decreased or were absent when the experiment was repeated (Experiment $3 b$ ).

775 It is possible that adult matching employs somewhat different strategies in comparison with

776 infant matching, which might explain why performance associations were notably lower or

777 absent. However, the low correlations can also be explained by the generally low performance on

778 the infant tasks. The low accuracy introduces restricted range issues and also raises the

779 possibility that much of the variability in performance is due to noise. As such, further work is

780 recommended before any conclusions can be drawn regarding the overlap in strategies/abilities

781 across the tasks.

782 We note that the majority of participants in Experiments 1 and 2 were women. Previous

783 research has shown that women, but not men, demonstrate an own-sex advantage on match trials,

784 and it also seems that women perform better than men on mismatch trials depicting either sex of

785 face (Megreya, Bindemann, \& Havard, 2011). Whether women show higher levels of accuracy

786 with infant and child face matching is currently unknown and future work might consider this

787 question further.

788 While previous research suggests that experience with infants may result in improved

789 infant face recognition (Cassia, Picozzi, Kuefner, \& Casati, 2009), we were unable to test the

790 idea that infant face matching would also be easier with increased experience. Unfortunately, the 
791 majority of undergraduate university students have minimal experience with infants. We might

792 predict, for example, that nursery school teachers, parents of young children, and midwives, may

793 all show higher levels of performance in comparison with our sample. However, we would still

794 expect lower levels for infant than for adult face matching, even in these populations.

795 Importantly though, evidence suggests that the quality of exposure may be crucial (Yovel et al., 796 2012), with improvement found only when people are required to individuate faces of a

797 particular category. Therefore, further research might consider the possibility of training through

798 individuation of infants/children in order to explore whether this may increase performance with 799 new identities.

800 It is interesting to consider how so-called "super-recognisers" (Russell, Duchaine, \&

801 Nakayama, 2009) might perform on our tasks of infant and child face matching. While these

802 individuals are remarkably good at both recognition and matching with adult faces (Bobak,

803 Hancock, \& Bate, 2016), researchers have yet to determine how well they perform with other 804 populations (e.g., infants or other-race faces). Anecdotally, some White super-recognisers have 805 reported being better with Black faces, although this may be the result of extensive 806 experience/contact with Black criminals and suspects (Davis, Jansari, \& Lander, 2013). If so, we 807 predict that super-recognisers would perform no better than the general population with our 808 current tasks.

809

810 Conclusion

811

812 Our experiments represent the first focussed investigation of the utility of infant and child

813 facial photographs for use in identification. Performance in both infant-infant and infant-child 
814 matching tasks was noticeably lower than with typical adult face matching tests. Despite the 815 randomised pairing of identities, the low levels of accuracy we observed were only minimally 816 aided (if at all) by the availability of sex category information. Taken together, and in 817 combination with previous work (White et al., 2015), our results suggest that such low levels of 818 accuracy mean infant and child facial photographs are ineffective for use in real-world 819 identification, and so alternative methods should be considered.

820

821 References

822

823 Bobak, A. K., Dowsett, A. J., \& Bate, S. (2016). Solving the border control problem: Evidence 824 of enhanced face matching in individuals with extraordinary face recognition skills. PLoS 825 ONE, 11(2), e0148148.

826

Bobak, A. K., Hancock, P. J. B., \& Bate, S. (2016). Super-recognisers in action: Evidence from 827 face-matching and face memory tasks. Applied Cognitive Psychology, 30(1), 81-91.

828

829

830

831

832

833

834 Burton, A. M., White, D., \& McNeill, A. (2010). The Glasgow face matching test. Behavior 835 Research Methods, 42(1), 286-291. 
836 Cassia, V. M., Picozzi, M., Kuefner, D., \& Casati, M. (2009). Why mix-ups don't happen in the

837

838

839

840

841

842

843

844

845

846

847

848

849

850

851

852

853

854

855

856 nursery: Evidence for an experience-based interpretation of the other-age effect. The Quarterly Journal of Experimental Psychology, 62(6), 1099-1107.

Chakravarty, M. M., Aleong, R., Leonard, G., Perron, M., Pike, G. B., Richer, L., ... \& Paus, T. (2011). Automated analysis of craniofacial morphology using magnetic resonance images. PLoS ONE, 6(5): e20241.

Chance, J. E., Goldstein, A. G., \& Andersen, B. (1986). Recognition memory for infant faces: An analog of the other-race effect. Bulletin of the Psychonomic Society, 24(4), 257-260.

Clutterbuck, R., \& Johnston, R. A. (2002). Exploring levels of face familiarity by using an indirect face-matching measure. Perception, 31, 985-994.

Clutterbuck, R., \& Johnston, R. A. (2004). Matching as an index of face familiarity. Visual Cognition, 11, 857-869.

Clutterbuck, R., \& Johnston, R. A. (2005). Demonstrating how unfamiliar faces become familiar using a face matching task. European Journal of Cognitive Psychology, 17, 97-116.

Davis, J. P., Jansari, A., \& Lander, K. (2013). 'I never forget a face!'. The Psychologist, 26(10), 726-729.

Dowsett, A. J., \& Burton, A. M. (2015). Unfamiliar face matching: Pairs out-perform individuals and provide a route to training. British Journal of Psychology, 106, 433-445.

Enlow, D. (1982). Handbook of facial growth. Philadelphia, PA: Saunders.

Estudillo, A. J., \& Bindemann, M. (2014). Generalization across view in face memory and face matching. i-Perception, 5, 589-601. 
857 Farkas, L. G., Posnick, J. C., Hreczko, T. M., \& Pron, G. E. (1992). Growth patterns of the 858 nasolabial region: A morphometric study. The Cleft Palate-Craniofacial Journal, 29(4), $859 \quad 318-324$.

860 Ferrario, V. F., Sforza, C., Serrao, G., Colombo, A., \& Ciusa, V. (1999). Soft tissue facial growth 861 and development as assessed by the three-dimensional computerized mesh diagram

862 863 analysis. American Journal of Orthodontics and Dentofacial Orthopedics, 116(2), 215226.

864 Fysh, M. C., \& Bindemann, M. (2018). The Kent face matching test. British Journal of 865 Psychology, 109(2), 219-231.

866 Hancock, P. J. B., Bruce, V., \& Burton, A. M. (2000). Recognition of unfamiliar faces. Trends in 867 Cognitive Sciences, 4(9), 330-337.

868 Harrison, V., \& Hole, G. J. (2009). Evidence for a contact-based explanation of the own-age bias 869 in face recognition. Psychonomic Bulletin \& Review, 16(2), 264-269.

870 Her Majesty’s (HM) Passport Office (n.d.). Rules for passport photos. Retrieved from 871 https://www.gov.uk/photos-for-passports/photo-requirements

872 Kaminski, G., Méary, D., Mermillod, M., \& Gentaz, E. (2011). Is it a he or a she? Behavioral 873 and computational approaches to sex categorization. Attention, Perception, \& 874 Psychophysics, 73(5), 1344-1349.

875 Kemp, R. I., Caon, A., Howard, M., \& Brooks, K. R. (2016). Improving unfamiliar face 876 877 matching by masking the external facial features. Applied Cognitive Psychology, 30, 622627.

878 Kemp, R., Towell, N., \& Pike, G. (1997). When seeing should not be believing: Photographs, 879 credit cards and fraud. Applied Cognitive Psychology, 11(3), 211-222. 
880 Kokje, E., Bindemann, M., \& Megreya, A. M. (2018). Cross-race correlations in the abilities to

881 match unfamiliar faces. Acta Psychologica, 185, 13-21.

882 Kramer, R. S. S., Manesi, Z., Towler, A., Reynolds, M. G., \& Burton, A. M. (2018). Familiarity 883 and within-person facial variability: The importance of the internal and external

884

885

886

887 888

889

890

891

892

893

894

895

896

897

898

899

900 features. Perception, 47(1), 3-15.

Kramer, R. S. S., \& Reynolds, M. G. (2018). Unfamiliar face matching with frontal and profile views. Perception, 47(4), 414-431.

Kramer, R. S. S., \& Ritchie, K. L. (2016). Disguising Superman: How glasses affect unfamiliar face matching. Applied Cognitive Psychology, 30(6), 841-845.

Megreya, A. M., Bindemann, M., \& Havard, C. (2011). Sex differences in unfamiliar face identification: Evidence from matching tasks. Acta Psychologica, 137(1), 83-89.

Megreya, A. M., \& Burton, A. M. (2006). Unfamiliar faces are not faces: Evidence from a matching task. Memory \& Cognition, 34(4), 865-876.

Megreya, A. M., \& Burton, A. M. (2007). Hits and false positives in face matching: A familiarity-based dissociation. Perception \& Psychophysics, 69(7), 1175-1184.

Megreya, A. M., \& Burton, A. M. (2008). Matching faces to photographs: Poor performance in eyewitness memory (without the memory). Journal of Experimental Psychology: Applied, 14(4), 364-372.

Megreya, A. M., Sandford, A., \& Burton, A. M. (2013). Matching face images taken on the same day or months apart: The limitations of photo ID. Applied Cognitive Psychology, 27(6), 700-706. 
901 Megreya, A. M., White, D., \& Burton, A. M. (2011). The other-race effect does not rely on 902 memory: Evidence from a matching task. The Quarterly Journal of Experimental 903 Psychology, 64(8), 1473-1483.

904 Meissner, C. A., \& Brigham, J. C. (2001). Thirty years of investigating the own-race bias in 905 memory for faces: A meta-analytic review. Psychology, Public Policy, and Law, 7(1), 3-

906 35.

907 Meissner, C. A., Susa, K. J., \& Ross, A. B. (2013). Can I see your passport please? Perceptual 908 discrimination of own- and other-race faces. Visual Cognition, 21(9-10), 1287-1305.

909 Porter, R. H., Cernoch, J. M., \& Balogh, R. D. (1984). Recognition of neonates by facial-visual $910 \quad$ characteristics. Pediatrics, 74(4), 501-504.

911 Rhodes, M. G., \& Anastasi, J. S. (2012). The own-age bias in face recognition: A meta-analytic 912 and theoretical review. Psychological Bulletin, 138(1), 146-174.

913 Robertson, D. J., Noyes, E., Dowsett, A. J., Jenkins, R., \& Burton, A. M. (2016). Face

914 recognition by Metropolitan Police super-recognisers. PLoS ONE, 11(2): e0150036.

915

916 $325(7378), 1446-1447$.

917 Russell, R., Duchaine, B., \& Nakayama, K. (2009). Super-recognizers: People with extraordinary 918 face recognition ability. Psychonomic Bulletin \& Review, 16(2), 252-257.

919 Seamon, J. G. (1982). Dynamic facial recognition: Examination of a natural phenomenon. 920 American Journal of Psychology, 95(3), 363-381.

921 Tskhay, K. O., \& Rule, N. O. (2016). People automatically extract infants' sex from faces. 922 Journal of Nonverbal Behavior, 40(4), 247-254. 
923 United Nations Office on Drugs and Crime (UNODC). (2016). Global report on trafficking in

924 persons. Retrieved from http://www.unodc.org/documents/data-and-

925 analysis/glotip/2016_Global_Report_on_Trafficking_in_Persons.pdf

926 U.S. Department of State. (2007). Trafficking in persons report. Retrieved from

927 https://www.state.gov/documents/organization/82902.pdf

928 Webb, R., Ayers, S., \& Endress, A. (2018). The City Infant Faces Database: A validated set of 929 infant facial expressions. Behavior Research Methods, 50(1), 151-159.

930 White, D., Dunn, J. D., Schmid, A. C., \& Kemp, R. I. (2015). Error rates in users of automatic 931 face recognition software. PLoS ONE, 10(10): e0139827.

932 White, D., Kemp, R. I., Jenkins, R., Matheson, M., \& Burton, A. M. (2014). Passport officers' 933 errors in face matching. PLoS ONE, 9(8): e103510.

934 Yadav, D., Singh, R., Vatsa, M., \& Noore, A. (2014). Recognizing age-separated face images: 935 Humans and machines. PLoS ONE, 9(12), e112234.

936 Yovel, G., Halsband, K., Pelleg, M., Farkash, N., Gal, B., \& Goshen-Gottstein, Y. (2012). Can

937 massive but passive exposure to faces contribute to face recognition abilities? Journal of 938 Experimental Psychology: Human Perception and Performance, 38(2), 285-289.

939

940 


\section{Figure captions}

942

943 Figure 1. Example match trial from Experiment 1. Both images A and B show the same infant.

944 Figure adapted from Webb et al. (2018) [CC BY 4.0].

945

946 Figure 2. Images illustrating a match trial in Experiment 2. The same child is shown at 8 months

947 (A) and 5 years old (B). (Copyright restrictions prevent publication of the original images.

948 Images shown here feature an identity who did not appear in the experiment. This person, now

949 aged 26, has given permission for her images to be reproduced here.) 


\section{Figure 1}

Example match trial from Experiment 1.

Both images A and B show the same infant. Figure adapted from Webb et al. (2018) [CC BY 4.0].
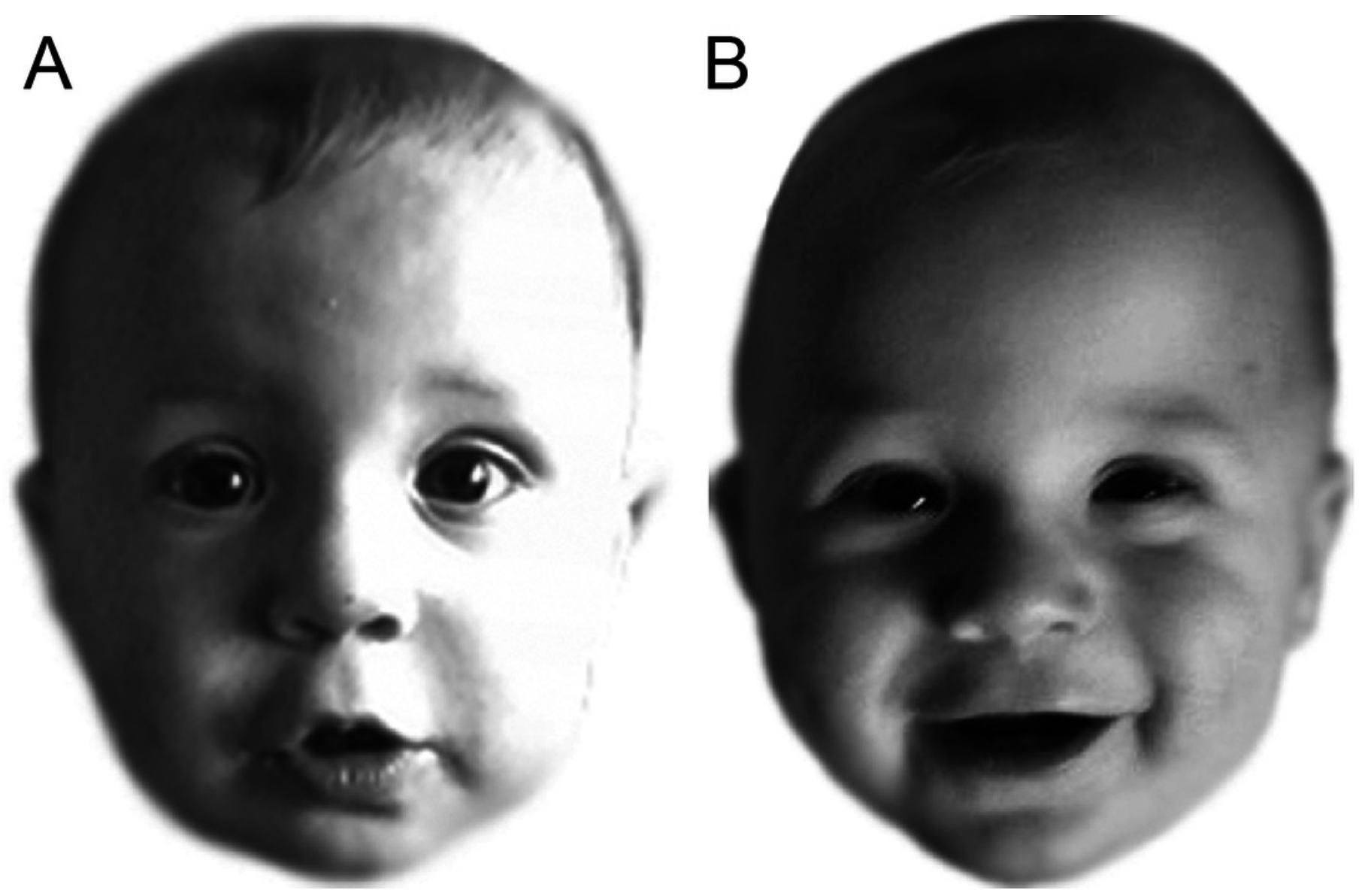


\section{Figure 2}

Images illustrating a match trial in Experiment 2.

The same child is shown at 8 months (A) and 5 years old (B). (Copyright restrictions prevent publication of the original images. Images shown here feature an identity who did not appear in the experiment. This person, now aged 26, has given permission for her images to be reproduced here.)

*Note: Auto Gamma Correction was used for the image. This only affects the reviewing manuscript. See original source image if needed for review.
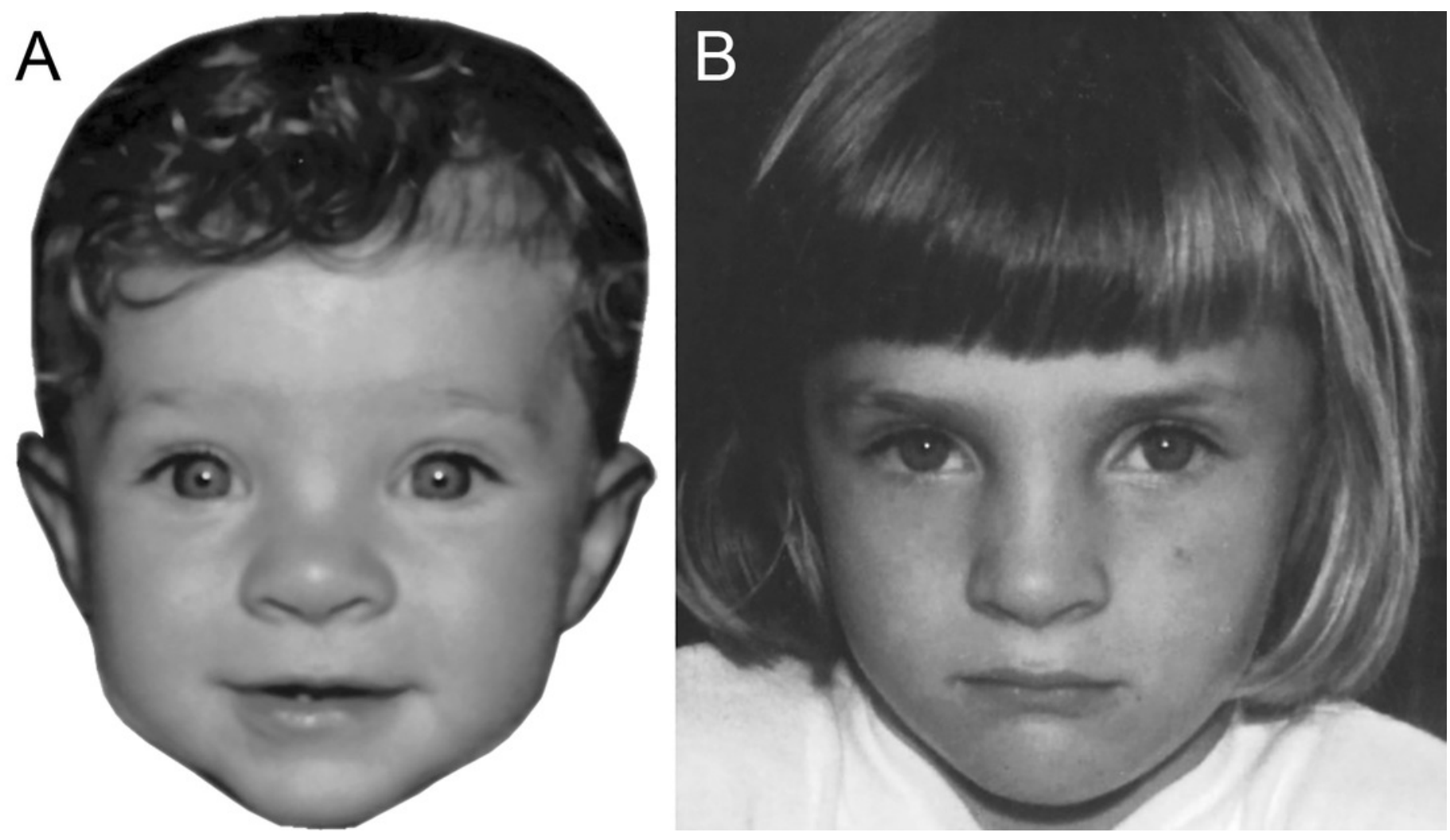


\section{Table $\mathbf{1}$ (on next page)}

A summary of the results for the current experiments and a benchmark face matching task.

GFMT = The Glasgow face matching test. Values presented are $M(S D)$. 
1 Table 1. A summary of the results for the current experiments and a benchmark face matching task.

\begin{tabular}{|c|c|c|c|c|c|c|}
\hline Source & Stimuli & $\%$ Overall & Hit Rate & False Alarm Rate & $d^{\prime}$ & $c$ \\
\hline Experiment 1 & 2 infant faces & $72.0(6.03)$ & $0.71(0.12)$ & $0.27(0.12)$ & $1.26(0.42)$ & $0.03(0.35)$ \\
\hline Experiment 2 & 1 infant +1 child face & $64.4(7.71)$ & $0.64(0.11)$ & $0.35(0.11)$ & $0.78(0.45)$ & $0.02(0.23)$ \\
\hline Experiment 3a & 2 infant faces & $56.4(7.04)$ & $0.47(0.16)$ & $0.34(0.17)$ & $0.37(0.43)$ & $0.27(0.44)$ \\
\hline Experiment 3a & 1 infant +1 child face & $52.9(8.62)$ & $0.54(0.15)$ & $0.49(0.17)$ & $0.15(0.48)$ & $-0.04(0.39)$ \\
\hline Experiment $3 \mathrm{a}$ & $\begin{array}{l}2 \text { adult faces (GFMT } \\
\text { short ver.) }\end{array}$ & $73.3(12.7)$ & $0.76(0.19)$ & $0.30(0.20)$ & $1.52(0.92)$ & $-0.11(0.52)$ \\
\hline Experiment $3 b$ & 2 infant faces & $55.7(7.09)$ & $0.44(0.21)$ & $0.33(0.21)$ & $0.34(0.42)$ & $0.36(0.62)$ \\
\hline Experiment $3 b$ & 1 infant +1 child face & $51.5(7.63)$ & $0.53(0.20)$ & $0.50(0.20)$ & $0.08(0.43)$ & $-0.02(0.57)$ \\
\hline Experiment $3 b$ & $\begin{array}{l}2 \text { adult faces (GFMT } \\
\text { short ver.) }\end{array}$ & $70.4(12.89)$ & $0.71(0.23)$ & $0.30(0.23)$ & $1.34(0.87)$ & $-0.01(0.69)$ \\
\hline Burton et al. (2010) & 2 adult faces (GFMT) & $89.9(7.3)$ & $0.92(0.08)$ & $0.12(0.11)$ & $2.91(0.83)$ & $-0.09(0.35)$ \\
\hline Bobak et al. (2016) & 2 adult faces (GFMT) & $87.4(5.26)$ & $0.91(0.07)$ & $0.12(0.08)$ & $2.82(0.73)$ & $-0.11(0.32)$ \\
\hline Burton et al. (2010) & $\begin{array}{l}2 \text { adult faces (GFMT } \\
\text { short ver.) }\end{array}$ & $81.2(9.4)$ & $0.80(0.14)$ & $0.18(0.12)$ & $2.04(0.84)$ & $0.06(0.39)$ \\
\hline
\end{tabular}

2 Note. GFMT $=$ The Glasgow face matching test. Values presented are $M(S D)$. 